\title{
EXPERIMENTAL CONTROL OF VARIABLE CAM TIMING ACTUATORS
}

\author{
Jonathan Chauvin* Nicolas Petit** \\ * Institut Français du Pétrole, 1 et 4 Avenue de Bois \\ Préau, 92852 Rueil Malmaison, France \\ jonathan. chauvin@ifp.fr \\ ** Centre Automatique et Systèmes, École des Mines de \\ Paris, \\ 60, bd St Michel, 75272 Paris, France
}

\begin{abstract}
In this paper, we propose a control scheme for the position of Variable Cam Timing (VCT) actuators. We take into account the observation by (Genç et al., 2001) that these actuators feature a significant nonlinearity, but we use a different approach. We perform a model reduction of mechanics, pressure, flows and balance equations. It serves to design an observer used in a motion planning strategy that is ultimately complemented by a tracking controller. Supportive vehicle experimental results are presented.
\end{abstract}

Keywords: Variable Cam Timing, actuator control, motion planning

\section{INTRODUCTION}

In several seminal papers (Gray, 1988; Ma, 1988; Leone et al., 1996; Stein and Galietti, 1995), it was demonstrated that optimization and realtime control of cam timing in an engine equipped with a dual equal camshaft timing phaser could help reduce the unburned hydrocarbons $(\mathrm{HC})$ and oxides of nitrogen $\left(\mathrm{NO}_{x}\right)$. In particular, by retarding the cam timing, a large part of combustion products, which would otherwise be expelled during the exhaust stroke, are retained in the cylinder during the subsequent intake stroke. This dilution of the mixture in the cylinder reduces $\mathrm{HC}$ and $\mathrm{NO}_{x}$ feedgas emissions. Nevertheless, retarding the cam timing have significant influence on the combustion stability. More details can be found in (Gray, 1988; Ma, 1988; Leone et al., 1996; Stein and Galietti, 1995; Stefanopoulou et al., 2000; Jankovic et al., 1998). Table 1 illustrates the sought after $\mathrm{NO}_{x}$ emission reduction when the Variable Cam Timing (VCT) actuators are used (more details can be found in (Le Solliec et al., 2007)). The VCT technology uses electron-

\begin{tabular}{|c|c||c|}
\hline $\mathrm{VCT}_{\text {int }}\left({ }^{\circ} \mathrm{CA}\right)$ & $\mathrm{VCT}_{\text {exh }}\left({ }^{\circ} \mathrm{CA}\right)$ & $\mathrm{NO}_{x}(\mathrm{ppm})$ \\
\hline 30 & 18 & 1302 \\
\hline 30 & 28 & 1079 \\
\hline 20 & 28 & 723 \\
\hline 10 & 28 & 302 \\
\hline 10 & 38 & 125 \\
\hline
\end{tabular}

Table 1. Experimental variation of VCT actuators (intake and exhaust) at fixed operating conditions (1500rpm, 30Nm).

$\mathrm{NO}_{x}$ pollutant emissions are lowered by retarding the cam timing.

ically controlled hydraulic actuators to adjust the inlet and exhaust valve timings to the crankshaft position. In the vast majority of the literature, VCT actuators have been treated as linear systems for which simple linear controllers for position control (Hathou et al., 2004; Stefanopoulou et al., 2000; Jankovic et al., 1998).

Yet, in (Genç et al., 2001), it was shown that VCT actuators have significant nonlinearities. The au- 
thors proposed a nonlinear model and a PID controller associated with a nonlinear function. Besides an input-output transport delay, an input static nonlinearity was identified in closed-loop and, eventually, inverted. Our approach is different. We desire to achieve fast and, very importantly, accurate transients. We propose an alternative model reduction obtained thanks to a singular perturbation approach (as used in (Eryilmaz and Wilson, 2001) and (Manhartsgruber, 2000)). Then, an open-loop strategy, designed on the basis of this simplified model, is proposed to steer the system from one steady state to another.

The paper is organized as follows. In Section 2, we propose a model of the VCT. This model is reduced to a second order nonlinear system using a singular perturbation approach. In Section 3, we develop a nonlinear state observer and prove its convergence. We propose a feedforward strategy and a feedback control law in Section 4. Implementation and experimental results are detailed in Section 5. Finally, conclusions and further development are exposed in Section 6 .

\section{MODELING OF THE VCT ACTUATOR}

\subsection{Physical modeling}

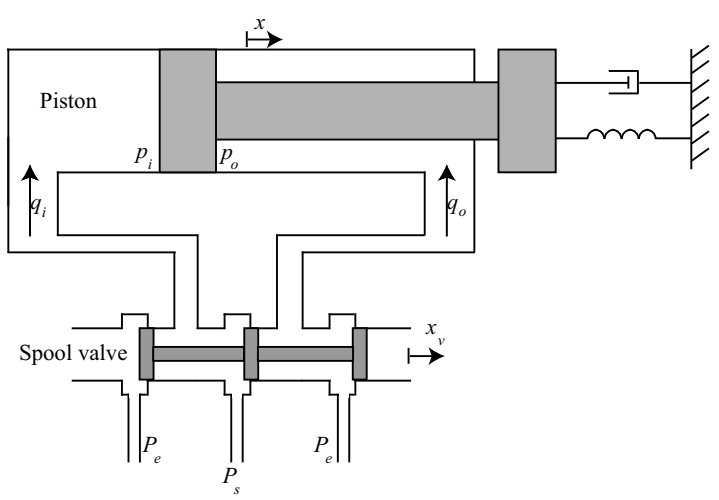

Figure 1. Variable Valve Timing actuator scheme.

The physical model of a VCT actuator is presented in Figure 1. A spool valve controls the pressure levels $p_{i}$ and $p_{o}$ through the displacement $x_{v}$. Noting $S$ the piston section area, the net force acting on the piston is $S\left(p_{i}-p_{o}\right)$. It determines the displacement $x$, which alters the valve timing. The piston is connected to one of the walls through a spring and a viscous damping (this is a classic modeling assumption see (Eryilmaz and Wilson, 2001)). Classically, the physical equations governing the system can be written under the form (deSilva, 1989)

$$
m_{p} \ddot{x}+b \dot{x}+k x=S\left(p_{i}-p_{o}\right)
$$

where $m_{p}$ is the mass of the piston, $b$ is the damping constant and $k$ is the spring stiffness. The numerical values in S.I. units are given in Table 2. The displacement $x_{v}$ is controlled by an electric motor whose input voltage $u$ can be freely chosen. Taking into account an extraneous input disturbance $x_{d}$ (standing for possible fluctuations in the engine oil temperature and pressure), the dynamics of the spool valve can be modelled as

$$
\tau \dot{x}_{v}+x_{v}=x_{d}+k_{v} u
$$

$x_{v}$ is strictly positive, i.e. there exists $\bar{x}_{v}>0 \mathrm{~s}$. t. $x_{v} \geq \bar{x}_{v}$, while $u$ is bounded, i.e. there exist $(\underline{u}, \bar{u})$ s.t. $\bar{u} \geq u \geq \underline{u}>0$. The flows $q_{i}$ and $q_{o}$ impacts on the pressures $p_{i}$ and $p_{o}$. An (oil) mass balance in both part of the chamber leads to

$$
\left\{\begin{array}{l}
\frac{V+S x}{\beta} \dot{p}_{i}=-S \dot{x}+q_{i}-\mu\left(p_{i}-p_{o}\right) \\
\frac{V-S x}{\beta} \dot{p}_{o}=S \dot{x}-q_{o}+\mu\left(p_{i}-p_{o}\right)
\end{array}\right.
$$

where the inlet and outlet (volumetric) flows $q_{i}$ and $q_{o}$ write

$$
\left\{\begin{array}{l}
q_{i}=c_{d} w x_{v} \sqrt{\frac{2}{\rho}\left(P_{s}-p_{i}\right)} \\
q_{o}=c_{d} w x_{v} \sqrt{\frac{2}{\rho}\left(p_{o}-P_{e}\right)}
\end{array}\right.
$$

Gathering (1)-(2)-(3)-(4), the system dynamics writes

$$
\left\{\begin{array}{c}
\ddot{x}=-\frac{b}{m_{p}} \dot{x}-\frac{k}{m_{p}} x+\frac{S}{m_{p}}\left(p_{i}-p_{o}\right) \\
\dot{x}_{v}=-\frac{1}{\tau}\left(x_{v}-x_{d}\right)+\frac{k_{v}}{\tau} u \\
\dot{p}_{i}=\frac{\beta}{V+S x}\left(-S \dot{x}+c_{d} w x_{v} \sqrt{\frac{2}{\rho}\left(P_{s}-p_{i}\right)}\right. \\
\left.-\mu\left(p_{i}-p_{o}\right)\right) \\
\dot{p}_{o}=\frac{\beta}{V-S x}\left(S \dot{x}-c_{d} w x_{v} \sqrt{\frac{2}{\rho}\left(p_{o}-P_{e}\right)}\right. \\
\left.+\mu\left(p_{i}-p_{o}\right)\right)
\end{array}\right.
$$

\begin{tabular}{|c|c||c|c|}
\hline Param. & Value & Param. & Value \\
\hline$b$ & 6 & $S$ & $6 e-4$ \\
$c_{d}$ & .67 & $V$ & $5 e-6$ \\
$m_{p}$ & .5 & $w$ & .02 \\
$k$ & $5 e 3$ & $\beta$ & 1.6 \\
$k_{v}$ & .02 & $\mu$ & $1 e-11$ \\
$P_{e}$ & $2 e 5$ & $\rho$ & 833 \\
$P_{s}$ & $2 e 7$ & $\tau$ & .005 \\
\hline
\end{tabular}

Table 2. Numerical value in S.I. units.

\subsection{Model simplification by singular perturbation}

The fifth order nonlinear system (5) accurately describes the dynamics of the system. However, 
one can notice that the pressure dynamics and the valve dynamics are much faster than the mechanical dynamics. Indeed, typically we have $\frac{V}{\beta} \simeq \frac{1}{200} S \simeq 3 e-6$ and $\tau=5 e-3$. This suggests to simplify these dynamics with a singular perturbation method (Khalil, 1992). Let $\epsilon \triangleq \frac{V}{\beta}$ be a scalar that represents all the small parameters to be neglected. The reference dynamics (5) has the form of the standard singularly perturbed system

$$
\left\{\begin{array}{r}
\dot{z}_{1}=\phi\left(z_{1}, z_{2}, u\right) \\
\epsilon \dot{z}_{2}=\psi\left(z_{1}, z_{2}, \epsilon\right)
\end{array}\right.
$$

where $z_{1} \triangleq\left[\begin{array}{lll}x & \dot{x} & x_{v}\end{array}\right]^{T}, z_{2} \triangleq\left[\begin{array}{ll}p_{i} & p_{o}\end{array}\right]^{T}$. Noting the time constants $T_{1}=\frac{V+S x}{\beta}=\mathcal{O}(\epsilon), T_{2}=$ $\frac{V-S x}{\beta}=\mathcal{O}(\epsilon)$ and $S=\mathcal{O}(\epsilon)$, i.e. $T_{1}=k_{1}(\epsilon) \epsilon$, $T_{2}=k_{2}(\epsilon) \epsilon$, and $S=k_{s}(\epsilon) \epsilon$ with $\lim _{\epsilon \rightarrow 0} k_{1}(\epsilon)=$ $\bar{k}_{1}>0, \lim _{\epsilon \rightarrow 0} k_{2}(\epsilon)=\bar{k}_{2}>0$, and $\lim _{\epsilon \rightarrow 0} k_{s}(\epsilon)=$ $\bar{k}_{s}>0$,we have

$$
\begin{aligned}
& \psi\left(z_{1}, z_{2}, \epsilon\right)= \\
& {\left[\begin{array}{l}
\frac{1}{k_{1}(\epsilon)}\left(-k_{s}(\epsilon) \dot{x}+\alpha\left(x_{v}\right) \sqrt{P_{s}-p_{i}}-\mu\left(p_{i}-p_{o}\right)\right) \\
\frac{1}{k_{2}(\epsilon)}\left(k_{s}(\epsilon) \dot{x}+\alpha\left(x_{v}\right) \sqrt{p_{o}-P_{e}}+\mu\left(p_{i}-p_{o}\right)\right)
\end{array}\right]}
\end{aligned}
$$

where $\alpha\left(x_{v}\right) \triangleq c_{d} w x_{v} \sqrt{\frac{2}{\rho}}$.

The equation $\psi\left(z_{1}, z_{2}, 0\right)=0$ has a unique root of interest $z_{2}=h\left(z_{1}\right)$. In details, it is

$$
\left\{\begin{aligned}
p_{i}+p_{o} & =P_{s}+P_{e} \\
\alpha\left(x_{v}\right)^{2}\left(P_{s}-p_{i}\right) & =\left(\mu\left(P_{s}-P_{e}\right)-2 \mu\left(P_{s}-p_{i}\right)\right)^{2}
\end{aligned}\right.
$$

Thus, the only feasible solution writes

$$
p_{i}=P_{s}-\bar{p}\left(x_{v}\right), \text { and } p_{o}=P_{e}+\bar{p}\left(x_{v}\right)
$$

where

$$
\bar{p}\left(x_{v}\right) \triangleq \frac{4 \gamma+\alpha\left(x_{v}\right)^{2}-\alpha\left(x_{s p}\right) \sqrt{\alpha\left(x_{v}\right)^{2}+8 \gamma}}{8 \mu^{2}}
$$

where $\gamma \triangleq \mu^{2}\left(P_{s}-P_{e}\right)$. To ensure the validity of the simplification, we can check the uniform stability of the Jacobian of $\psi$ (Kokotović et al., 1999)[Assumption 3.2 p11]. For that, we consider $\partial_{z_{2}} \psi_{\mid z_{2}=h\left(z_{1}\right)}$ and compute its eigenvalues

$$
\mu\left[\begin{array}{cc}
\partial_{z_{2}} \psi_{\mid z_{2}=h\left(z_{1}\right)}= & \\
\mu\left[\begin{array}{cc}
-1-\frac{\alpha\left(x_{v}\right)}{\mu \sqrt{P_{s}-h_{1}\left(z_{1}\right)}} & 1 \\
1 & -1-\frac{\alpha\left(x_{s p}\right)}{\mu \sqrt{h_{2}\left(z_{1}\right)-P_{e}}}
\end{array}\right]
\end{array}\right.
$$

Since $x_{v}$ is positively bounded, there exists $c>0$ such that $\mathcal{R} e\left(\partial_{z_{2}} \psi_{\mid z_{2}=h\left(z_{1}\right)}\right)<-c$. The reduced dynamics writes

$$
\left\{\begin{array}{l}
\dot{\bar{z}}_{1}=\phi\left(\bar{z}_{1}, h\left(\bar{z}_{1}\right), u\right) \\
\bar{z}_{2}=h\left(\bar{z}_{1}\right)
\end{array}\right.
$$

From (Khalil, 1992)[Th 11.1], the following proposition holds
Proposition 1. Consider the singularly perturbated system $(6)$ and $z_{2}=h\left(z_{1}\right)$ the isolated root of $\psi\left(z_{1}, z_{2}\right)=0$. There exists a positive constant $\epsilon^{\star}>\epsilon>0$ such that (6) possesses a unique trajectory $z_{1}(t, \epsilon), z_{2}(t, \epsilon)$, and

$$
\begin{gathered}
z_{1}(t, \epsilon)-\bar{z}_{1}(t)=\mathcal{O}(\epsilon) \\
z_{2}(t, \epsilon)-h\left(\bar{z}_{1}(t)\right)=\mathcal{O}(\epsilon)
\end{gathered}
$$

hold when $\epsilon<\epsilon^{\star}$.

By the same singular perturbation approach, we can approximate the stable spool position as $x_{v} \simeq$ $x_{d}+k_{s p} u$. Finally, these simplifications lead to consider only the mechanical dynamics of the piston, i.e.

$$
\ddot{x}=-\alpha_{1} x-\alpha_{2} \dot{x}+f\left(u-u_{0}\right)
$$

where $\alpha_{1} \triangleq \frac{k}{m_{p}}, \alpha_{2} \triangleq \frac{b}{m_{p}}, u_{0} \triangleq-\frac{x_{d}}{k_{v}}$, and,

$$
f\left(u-u_{0}\right) \triangleq \frac{S}{m_{p}}\left(P_{s}-P_{e}-2 \bar{p}\left(k_{s p}\left(u-u_{0}\right)\right)\right)
$$

It appears that $f$ is a strictly increasing nonlinear function. There exists $\underline{f}>0$ such that $f^{\prime} \geq \underline{f}$. Experimentally, this function can be identified around steady state position. Figure 2 shows the result of the identification we perform on a 4 cylinder downsized engine described in 5.2. To validate

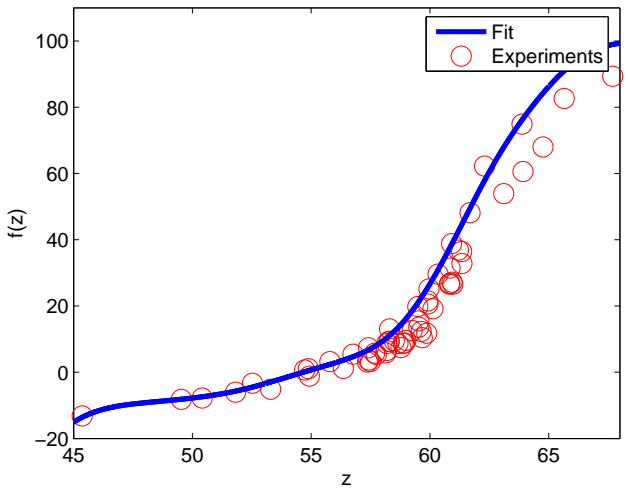

Figure 2. Experimentally identified function $f$.

the whole simplification process, we compare the results of the simplified model (8) against experimental results. This comparison is reported in Figure 3. The model catches the most significant dynamics and, as will be demonstrated in the next section, is relevant for control purposes.

\section{OBSERVER DESIGN}

Through the $k_{v}$ parameter, the value of $u_{0}$ depends on the oil temperature and pressure, and on the engine speed. Since the control strategy we propose relies on this value, we need to estimate it. For that purpose, we design an observer. The input signal $u_{0}$ is considered to be slowly varying 


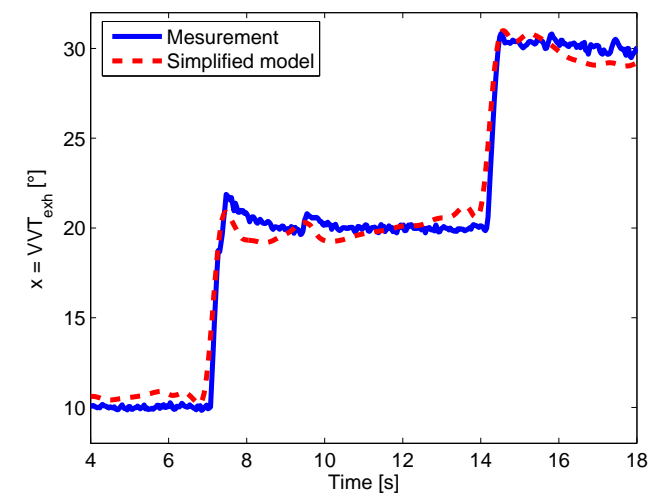

Figure 3. Comparison of the simplified model (8) against experimental measurements.

in $(8)$, i.e. $\dot{u}_{0}=0$. The reference nonlinear system that serves for the observer design is

$$
\left\{\begin{aligned}
\dot{x} & =v \\
\dot{v} & =-\alpha_{1} x-\alpha_{2} v+f\left(u-u_{0}\right), \quad y=x \\
\dot{u}_{0} & =0
\end{aligned}\right.
$$

\subsection{Observer definition}

To observe (9), we propose the following observer

$$
\left\{\begin{array}{l}
\dot{\hat{x}}=\hat{v}-L_{x} \alpha_{2}(\hat{x}-y) \\
\dot{\hat{v}}=-\alpha_{1} \hat{x}-\alpha_{2} \hat{v}+f\left(u-\hat{u}_{0}\right)-L_{v} \alpha_{1}(\hat{x}-y) \\
\dot{\hat{u}}_{0}=-\frac{L_{u}}{f^{\prime}\left(u-\hat{u}_{0}\right)}(\hat{x}-y)
\end{array}\right.
$$

where $L_{x}, L_{v}$ and $L_{u}$ are strictly positive parameters. Then, the error dynamics writes

$$
\left\{\begin{array}{l}
\dot{\tilde{X}}=A \tilde{X}+B \delta\left(\tilde{u}_{0}\right) \\
\dot{\tilde{u}}_{0}=-\frac{L_{u}}{\delta^{\prime}\left(\tilde{u}_{0}\right)} C \tilde{X}
\end{array}\right.
$$

where $\tilde{X} \triangleq\left[\begin{array}{c}\tilde{x} \\ \tilde{v}\end{array}\right], A \triangleq\left[\begin{array}{cc}-l_{x} \alpha_{2} & 1 \\ -\left(l_{v}+1\right) \alpha_{1} & -\alpha_{2}\end{array}\right], B \triangleq$ $\left[\begin{array}{l}0 \\ 1\end{array}\right]$ and $C \triangleq\left[\begin{array}{ll}1 & 0\end{array}\right]$, and $\delta\left(\tilde{u}_{0}\right) \triangleq f\left(u-u_{0}+\tilde{u}_{0}\right)-$ $f\left(u-u_{0}\right)$.

\subsection{Convergence analysis}

The proof proceeds from a Lyapunov approach. To get some insight into the derivation of a Lyapunov function, one can consider, which is obviously wrong, that the system is linear, i.e. $\delta\left(\tilde{u}_{0}\right)=b \tilde{u}_{0}$. In that scenario, stability would be guaranteed because the matrix $\left[\begin{array}{cc}A & B \\ -L_{u} C & 0\end{array}\right]$ is stable. Thus, there would exist a symmetric positive definite matrix $P$ solution of the Lyapunov equation

$$
P\left[\begin{array}{cc}
A & B \\
-L_{u} C & 0
\end{array}\right]+\left[\begin{array}{cc}
A & B \\
-L_{u} C & 0
\end{array}\right]^{T} P=-I
$$

In this case, a Lyapunov function is

$$
V\left(\tilde{X}, \tilde{u}_{0}\right)=\left[\begin{array}{cc}
\tilde{X} & \tilde{u}_{0}
\end{array}\right] P\left[\begin{array}{cc}
\tilde{X} & \tilde{u}_{0}
\end{array}\right]^{T}
$$

This inspires us in the nonlinear case. We keep the same idea and use as Lyapunov function

$$
V\left(\tilde{X}, \tilde{u}_{0}\right)=\left[\begin{array}{ll}
\tilde{X} & \delta\left(\tilde{u}_{0}\right)
\end{array}\right] P\left[\begin{array}{ll}
\tilde{X} & \delta\left(\tilde{u}_{0}\right)
\end{array}\right]^{T}
$$

Again, $V$ is positive definite, and we have $\dot{V}=$ $-\tilde{X}^{T} \tilde{X}-\delta\left(\tilde{u}_{0}\right)^{2} \leq 0 . V$ is then a Lyapunov function. From Lyapunov's theorem (see for instance (Khalil, 1992) Theorem 4.2), the following proposition holds

Proposition 2. The error dynamics (11) (the error between the reference system (9) and the observer (10)) converges towards 0 as $t \rightarrow \infty$.

\section{CONTROLLER DESIGN}

We now focus on the controller design. We proceed in two steps. First, we design an open loop controller, and then, we complement it with a tracking feedback controller.

\subsection{Feedforward design}

Set points The driver's request considered here is the accelerator position. First, taking into account the gear box configuration, this request is turned into a torque control objective under the form of an IMEP (Indicated Mean Effective Pressure) set point. Then, the set points for the VCT are inversely given by experimentally calibrated static maps on the $\left(I M E P^{\mathrm{sp}}, N_{e}\right)$ operating range. The engine speed $N_{e}$ is not modelled but directly measured. $x^{\mathrm{sp}}$ is defined as $x^{\mathrm{sp}} \triangleq$ $f_{V C T}\left(I M E P^{\mathrm{sp}}, N_{e}\right)$.

Motion planning Because $I M E P^{\mathrm{sp}}$ is arbitrarily specified by the driver, $t \mapsto x^{\mathrm{sp}}(t)$ may not be smooth nor monotonous. These signals must be filtered to correspond to feasible trajectories of (8). This can be done by many methods (including filtering with tunable transfer functions). Here, we propose the following approach that, among several properties, is easy to handle in a convergence analysis process ${ }^{1}$. It addresses only the case of transients from one steady state to another. From a current steady state $\underline{x}$ to a target $\bar{x}$ an interpolation formula is used. Note $T$ a positive constant, let

$$
\phi(t, T)=\left\{\begin{array}{cl}
0 & \text { for } 0 \geq t \\
\left(\frac{t}{T}\right)^{2}\left(3-2 \frac{t}{T}\right) & \text { for } 0 \leq t \leq T \\
1 & \text { for } T \leq t
\end{array}\right.
$$

\footnotetext{
1 One can refer to (Chauvin et al., 2006) for a similar analysis on the airpath of a Diesel HCCI engine.
} 
The considered interpolation is

$$
x^{\mathrm{mp}}(t)=\underline{x}+(\bar{x}-\underline{x}) \phi(t, T)
$$

Model inversion System (8) is fully actuated and invertible. Thus, an analytic expression of the input can be derived from the state variables and their first derivatives histories. Namely $u \triangleq$ $g\left(x, \dot{x}, \ddot{x}, u_{0}\right)$

$$
u=u_{0}+f^{-1}\left(\ddot{x}+\alpha_{1} x+\alpha_{2} \dot{x}\right)
$$

The unique open-loop control law $\left(u^{\mathrm{mp}}\right)$ corresponding to any desired $\left(x^{\mathrm{mp}}\right)$ trajectory (defined by formulas (13)) is

$$
u^{\mathrm{mp}}=g\left(x^{\mathrm{mp}}, \dot{x}^{\mathrm{mp}}, \ddot{x}^{\mathrm{mp}}, u_{0}\right)
$$

\subsection{Feedback design}

The previously defined reference (13) must be tracked as fast as possible and disturbances must be accounted for. For that purpose and to provide robustness, we consider a feedback controller. Equivalently to $u$, we consider $v \triangleq f\left(u-u_{0}\right)$ as control. Then, we use a LQR controller to optimize the following performance index

$\int_{0}^{+\infty}\left[r_{x}\left(x-x^{\mathrm{mp}}\right)^{2}+r_{v}\left(\dot{x}-\dot{x}^{\mathrm{mp}}\right)^{2}+\left(v-v^{\mathrm{mp}}\right)^{2}\right] d t$ where $\left(r_{x}, r_{v}\right) \in\left(\mathbb{R}^{+}\right)^{2}$ are the tuning gains under the linear dynamics $\ddot{x}=-\alpha_{1} x-\alpha_{2} \dot{x}+v$. This infinite horizon problem yields constant controller gains $\left(k_{x}, k_{v}\right)$. The control writes

$u(t)=u_{0}+f^{-1}\left(f\left(u^{\mathrm{mp}}\right)-k_{x}\left(x-x^{\mathrm{mp}}\right)-k_{v}\left(\dot{x}-\dot{x}^{\mathrm{mp}}\right)\right)$

Details of implementation are given in the next section.

\section{EXPERIMENTAL RESULTS}

\subsection{Control scheme}

The global control strategy combines the observer (10), and the controller (16). It is summarized in Figure 4. The "VCT observer" block is the implementation of observer (10). This block gives an estimation of the position and the velocity of the VCT actuator. It also estimates the unknown input $u_{0}$. The "VCT dynamics inversion" block implements the motion planning open loop control strategy (15) where $u_{0}$ is substituted with $u_{0}$. The closed loop controller (16) appears in the "VCT controller" block to provide accuracy and robustness.

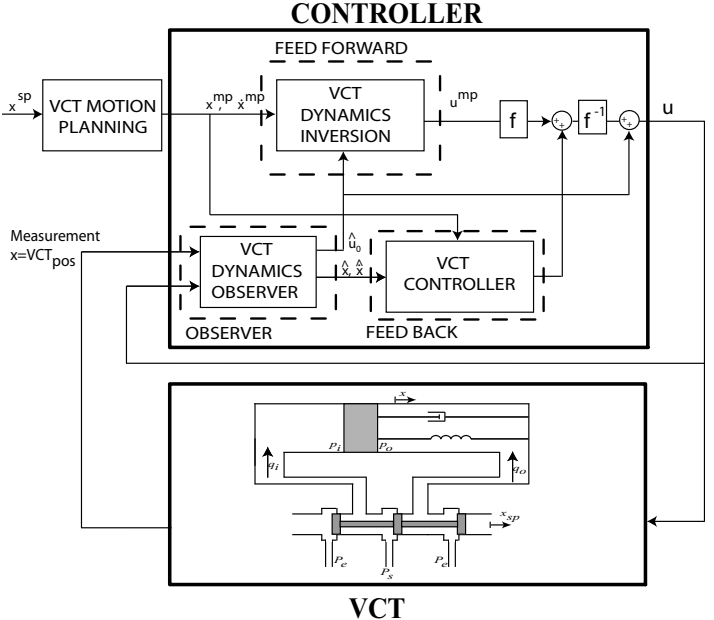

Figure 4. Implementation of the Variable Valve Timing actuator control scheme.

\subsection{Engine set-up}

We now briefly discuss the engine we conduct experimentations on, and, more generally, the downsizing context. The downsizing technique aims at replacing a given engine with one with smaller displacement volume without loss of performance or efficiency. At IFP, we achieve this by combining turbocharging, homogeneous direct injection and variable cam timing on both camshafts. A twinscroll turbine is used to maximize kinetic energy recovery through the strong pulsating exhaust pressures which are accentuated by small exhaust manifolds (Pagot et al., 2002). The main characteristics of the engine are:

- Four cylinder SI engine.

- Waste-gate turbocharger with twin-scroll turbine.

- Homogeneous direct gasoline injection.

- Variable valve timing on intake and exhaust camshafts (valve lift invariable).

- Stroke x Bore: 93 x $82.7 \mathrm{~mm}$.

- Compression ratio: $10.5: 1$.

Experimental results were obtained with this engine fitted in a Renault VelSatis vehicle.

\subsection{Experimental results}

At fixed engine speed First, we present results around steady state. Only the (exhaust) VCT setpoint at idle speed 900rpm varies. On this test, we compare the proposed control scheme and a PID controller are fully tuned. Figure 5 presents the VCT setpoint (solid-blue) and the experimental results with the two controllers: the observer/controller (dashdot green) and the reference PID (dotted red).

Besides these plots which clearly stress the superiority of the proposed method over the PID, 

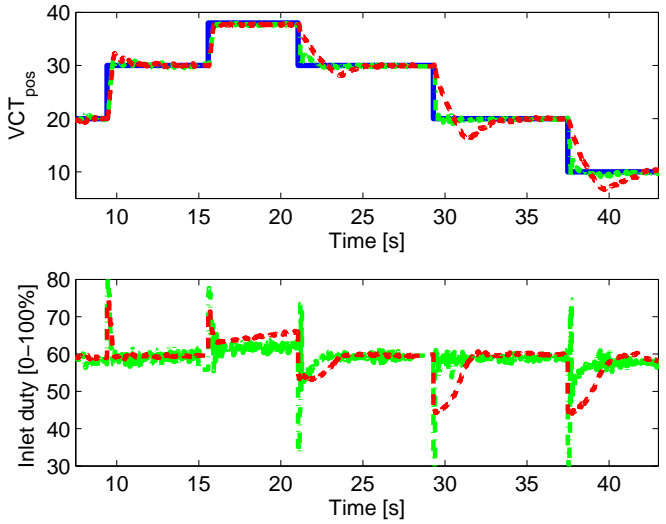

Figure 5. Experimental VCT control around idle speed (900rpm). Top: VCT setpoint $\left(x^{s p}\right)$ (solid blue), VCT position $(x)$ for the proposed controller (dashdot green), and reference PID (dotted red). Bottom: Control $u$ for the proposed controller (dashdot green), and reference PID (dotted red).

\begin{tabular}{|c||c|c||c|c|}
\hline \multicolumn{1}{|c||}{ Transient } & \multicolumn{2}{c||}{ PID } & \multicolumn{2}{c|}{ Proposed Controller } \\
\hline \hline & $\tau_{2 \%}$ & $\Delta$ & $\tau_{2 \%}$ & $\Delta$ \\
\hline \hline $20 \rightarrow 30$ & 2.31 & 23.0 & 2.16 & 8.83 \\
\hline $30 \rightarrow 40$ & 0.44 & 0 & 0.42 & 0 \\
\hline $40 \rightarrow 30$ & 4.18 & 24.1 & 2.05 & 5.6 \\
\hline $30 \rightarrow 20$ & 5.00 & 37.7 & 1.73 & 9.8 \\
\hline $20 \rightarrow 10$ & 5.43 & 34.0 & 2.99 & 11.3 \\
\hline
\end{tabular}

Table 3. Experimental results: Comparison between the PID controller and the proposed controller.

we wish to obtain numerical comparisons. We compute

- $\tau_{2 \%}$ : the time needed to get into the $\pm 2 \%$ band around the setpoint.

- $\Delta$ : the overshoot in $\%$.

Several transients results are reported in Table 3. The proposed controller is much faster than the PID controller. Overshoot is much reduced.

Under driving operating conditions Finally, we use this controller under driving operating conditions. Figure 6 shows the setpoint and the position of the exhaust VCT during a part of the European cycle. The operating conditions of this test (engine and vehicle speed) are presented at the bottom of the figure.

Results are very good. The VCT position setpoint trajectory is well tracked. Compared to the reference PID, the variance of the error between the position and its setpoint is divided by five (typically, $\operatorname{var}\left(V C T_{s p}-V C T_{m}\right)$ approximatively equals 7 with a PID and 1.35 with the proposed observer/controller).
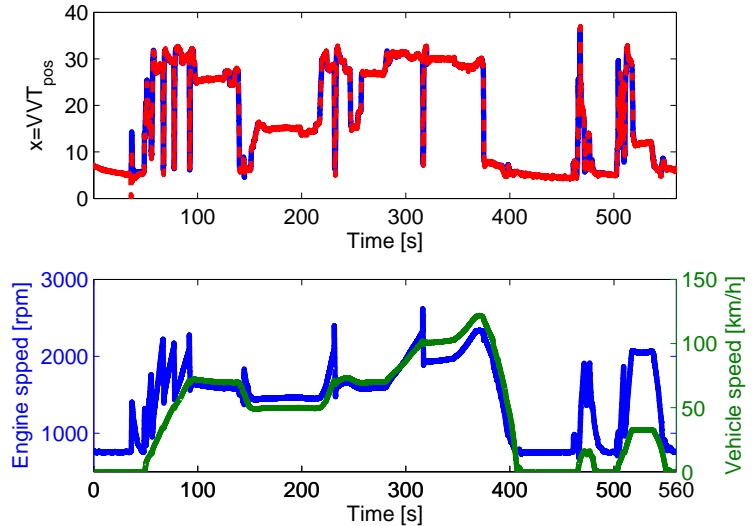

Figure 6. Experimental VCT control on the vehicle during a part of the European cycle. Top: Setpoint (solid blue), proposed controller (dotted red). Bottom: Operating conditions (Engine speed (rpm) and vehicle speed $(\mathrm{km} / \mathrm{h}))$.

\section{CONCLUSION}

In this paper, we propose an observer/controller structure for the management of VCT actuators displacement. We prove that we can derive a simple nonlinear model from the physical equations through a singular perturbation analysis. Experimental results prove the relevance of this control structure. Compared against a standard PID, we obtain a faster response with a low overshoot. Finally, this strategy is experimentally tested on a prototype vehicle. Results stress the performance of this approach. Under realistic driving conditions, it is possible to reduce by a factor of 5 the variance of the error between the reference and the VCT position. It appears that, while a modelling approach differs from the technique advocated by (Genç et al., 2001), we come to the same conclusion: it is interesting and not too difficult, to take the nonlinear nature of the VCT actuator into account (at least, the input nonlinearity that we identified).

\section{REFERENCES}

Chauvin, J., G. Corde, N. Petit and P. Rouchon (2006). Experimental motion planning in airpath control for HCCI engine. In: Proc. of the American Control Conference.

deSilva, C.L. (1989). Control Sensors and Actuators. Prentice Hall.

Eryilmaz, B. and B. Wilson (2001). Improved tracking control of hydraulic systems. Journal of Dynamic Systems, Measurement, and Control 123, 457-462.

Genç, A., K. Glover and R. Ford (2001). Nonlinear control of hydraulic actuators in variable cam timing engines. In: International IFAC 
Workshop on Modeling, Emissions and Control in Automotive Engines.

Gray, C. (1988). A review of variable engine valve timing. In: Proc. of the SAE Conference. number 880386.

Hathou, J.-P., J. Ahmed and A. Kojić (2004). Reduced order modeling and control of an electrohydrolic valve system. In: International IFAC Symposium "Advances in Automotive Control".

Jankovic, M., F. Frischmuth, A. Stefanopoulou and Cook J. (1998). Torque management of engines with variable cam timing. Proc. of the IEEE Control Systems Magazine 18, 34-42.

Khalil, H. (1992). Nonlinear Systems. PrenticeHall, Inc.

Kokotović, P., O. O'Reilly and H. Khalil (1999). Singular Perturbation Methods in Control: Analysis and Design. Soc. for Industrial \& Applied Math.

Le Solliec, G., F. Le Berr, G. Colin and G. Corde (2007). Downsized SI engine control : A torque-based design from simulation to vehicle. In: Proc. of the SAE Conference. number 2007-01-1506.

Leone, T.G., E.J. Christenson and R.A. Stein (1996). Comparison of variable camshaft timing strategies at part load. In: Proc. of the SAE Conference. number 960584.

Ma, T. H. (1988). Effects of variable engine valve timing on fuel economy. In: Proc. of the $S A E$ Conference. number 880390.

Manhartsgruber, B. (2000). Application of singular perturbation theory to hydraulic servo drives - system analysis and control design. In: 1st FPNI PhD Symposium Hamburg.

Pagot, A., G. Monnier, H. Strobach, D. Jeckel and Petitjean (2002). Turbocharging application on a gasoline direct injection engine. In: $A u$ fladetechnische Konferenz.

Stefanopoulou, A., J. Freudenberg and J. Grizzle (2000). Analysis and control of transient torque response in engines with internal exhaust gas recirculation. IEEE Transactions on Control System Technology 8(2), 23-34.

Stein, R.A. and T.G. Galietti, K.M. an Leone (1995). Dual equal VCT - a variable camshaft timing strategy for improved fuel economy and emissions. In: Proc. of the SAE Conference. number 950975. 